\title{
Editorial
}

\section{(Par-)enterale Ernährung - Zusammen sind sie stark!}

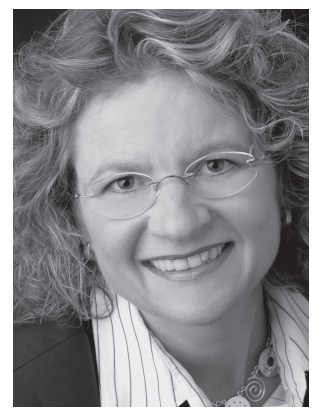

Essen und Trinken - für die einen ein Hochgenuss, der mit besten Zutaten und unter hohem Zeitaufwand zelebriert wird, für viele allerdings eine Beschäftigung, die so nebenbei abläuft. Ganz anders stellt sich die Situation bei Patienten dar, die sich nicht mehr auf normalem Wege ernähren können, da krankheitsbedingt das Schlucken, Transportieren oder Verdauen der Nahrung nicht mehr oder nur noch eingeschränkt möglich ist. Neben der Behandlung der zugrunde liegenden Erkrankung und den häufig damit verbundenen Einschränkungen bestimmt die Beschäftigung mit der ausreichenden Nahrungsaufnahme zu einem hohen Anteil den Tagesablauf. Plötzlich ist man auf verschiedene Gerätschaften, flüssige Nahrung statt intakter Lebensmittel und auch auf Personen angewiesen, die einem dabei helfen, ein menschliches Grundbedürfnis zu decken.

Die in dieser Situation infrage kommenden Ernährungsformen werden als klinische oder künstliche Ernährung bezeichnet und umfassen die enterale und parenterale Ernährung. Die enterale Ernährung, im Volksmund auch häufig als Astronautenkost bezeichnet, wird verwendet, solange der Verdauungstrakt noch funktionstüchtig ist und Nährstoffe resorbieren kann. Ist dies nicht mehr möglich, können Nährstoffe unter Umgehung des Verdauungstraktes intravenös appliziert werden. Die enterale und die parenterale Ernährung schließen einander nicht aus, sondern haben jeweils ihre Indikation und werden häufig auch kombiniert verwendet.
Entscheidend bei der Auswahl der jeweiligen Ernährungsform ist die Beantwortung der Frage, auf welchem Weg es am besten gelingt, den jeweiligen Nährstoffbedarf zu decken. Denn es gilt, eine Mangelernährung zu vermeiden, da Lebensqualität, Regenerierung und Genesung stark vom Ernährungszustand beeinflusst werden.

In der Regel wird der Patient in der Klinik auf die entsprechende (par-)enterale Ernährung eingestellt und führt diese dann zu Hause fort. Die häusliche Versorgung mit klinischer Ernährung stellt eine enorme Verbesserung der Lebensqualität dar. Vor allem im Bereich der parenteralen Ernährung bedeutet dies einen gewaltigen Fortschritt, denn die Durchführung im häuslichen Umfeld war früher undenkbar, heute ist sie - bei der entsprechenden Schulung und Betreuung alltäglich.

Wichtig für eine optimale Ernährungstherapie ist die interdisziplinäre Herangehensweise. Im Idealfall erfolgt eine Behandlung in enger Kooperation von Ernährungs- und Pflegefachkräften, Ärzten, Logopäden und anderen therapeutischen Berufsgruppen, die sich gegenseitig unterstützen und ergänzen. Es ist zu wünschen, dass sich die bereits begonnene Etablierung von multiprofessionellen Ernährungsteams weiter verbreitet.

\section{Dr. Bettina Dörr \\ Dipl.-Oecotrophologin \\ München}

